PROCEEDINGS OF THE

AMERICAN MATHEMATICAL SOCIETY

Volume 139, Number 2, February 2011, Pages 485-490

S 0002-9939(2010)10476-8

Article electronically published on July 12, 2010

\title{
IDEAL-TRIANGULARIZABILITY OF NIL-ALGEBRAS GENERATED BY POSITIVE OPERATORS
}

\author{
MARKO KANDIĆ
}

(Communicated by Nigel J. Kalton)

\begin{abstract}
R. Drnovšek, D. Kokol-Bukovšek, L. Livshits, G. MacDonald, M. Omladič, and H. Radjavi constructed an irreducible set of positive nilpotent operators on $L^{p}[0,1)$ which is closed under multiplication, addition and multiplication by positive real scalars with the property that any finite subset is ideal-triangularizable. In this paper we prove the following:

(1) every algebra of nilpotent operators which is generated by a set of positive operators on a Banach lattice is ideal-triangularizable whenever the nilpotency index of its operators is bounded;

(2) every finite subset of an algebra of nilpotent operators which is generated by a set of positive operators on a Banach lattice is ideal-triangularizable.
\end{abstract}

\section{INTRODUCTION AND PRELIMINARIES}

Let $E$ be a Banach lattice. By an operator on $E$ we mean a continuous linear transformation from $E$ into itself. An operator $T$ is called positive whenever $T x \in$ $E^{+}$for all $x \in E^{+}$. For the terminology not explained in the text we refer the reader to the books [10] and [14].

A family $\mathcal{F}$ of operators on $E$ is said to be reducible if there exists a nontrivial closed subspace of $E$ that is invariant under every member of $\mathcal{F}$. Otherwise, we say that $\mathcal{F}$ is irreducible. If there exists a maximal subspace chain (i.e., a maximal totally ordered set of closed subspaces) whose elements are invariant under every member of $\mathcal{F}$, then $\mathcal{F}$ is said to be triangularizable. A family $\mathcal{F}$ of operators on $E$ is said to be ideal-reducible if there exists a nontrivial closed ideal of $E$ which is invariant under every operator in $\mathcal{F}$. Otherwise, we say that $\mathcal{F}$ is ideal-irreducible.

A subset $\mathcal{I}$ of a semigroup $\mathcal{S}$ is said to be a semigroup ideal if $S T$ and $T S$ belong to $\mathcal{I}$ for all $S \in \mathcal{S}$ and $T \in \mathcal{I}$. For a subset $\mathcal{F}$ of a semigroup $\mathcal{S}$ we define $\mathcal{F}^{n}$ as the set of all products of at least $n$ elements of $\mathcal{F}$, and by $\operatorname{sg}(\mathcal{F})$ we denote the semigroup generated by $\mathcal{F}$. It is obvious that for each $n \in \mathbb{N}$ the set $\mathcal{F}^{n}$ is a semigroup ideal in $\operatorname{sg}(\mathcal{F})$.

The following proposition, which was proved in [3, is very useful in determining if a given semigroup of positive operators is ideal-reducible.

Received by the editors December 17, 2009 and, in revised form, March 2, 2010.

2010 Mathematics Subject Classification. Primary 47A15, 47B65; Secondary 16N40.

Key words and phrases. Banach lattices, positive operators, operator semigroups, idealreducibility, ideal-triangularizability, nilpotent algebras, nil-algebras.

This work was supported by the Slovenian Research Agency.

(C)2010 American Mathematical Society Reverts to public domain 28 years from publication 
Proposition 1.1. Let $E$ be a normed Riesz space, and let $\mathcal{S}$ be a nonzero semigroup of positive operators on $E$. The following statements are equivalent:

(1) $\mathcal{S}$ is ideal-reducible;

(2) there exist a nonzero positive functional $\varphi \in E^{*}$ and a nonzero positive vector $f \in E$ such that $\varphi(\mathcal{S} f)=\{0\}$;

(3) there exist nonzero positive operators $A$ and $B$ on $E$ such that $A \mathcal{S} B=\{0\}$;

(4) some nonzero semigroup ideal of $\mathcal{S}$ is ideal-reducible.

A family $\mathcal{F}$ is ideal-triangularizable if there is a chain $\mathcal{C}$ that is maximal as a chain of closed ideals of $E$ and that has the property that every ideal in $\mathcal{C}$ is invariant under all the operators in $\mathcal{F}$. Any such chain of closed ideals is said to be an ideal-triangularizing chain for the family $\mathcal{F}$. By [2, Proposition 1.2], every maximal chain of closed ideals of a Banach lattice is also maximal as a chain of closed subspaces of a Banach space. Thus, an ideal-triangularizable family of operators is also triangularizable. The notion of ideal-triangularizability was introduced by Jahandideh in $[9$.

Let $\mathcal{F}$ be a family of operators on $E$, and let $I$ and $J$ be closed ideals of $E$ satisfying $J \subseteq I$ that are invariant under every member of $\mathcal{F}$. Then $\mathcal{F}$ induces a family $\hat{\mathcal{F}}$ of operators on the quotient Banach lattice $I / J$ as follows. For each $T \in \mathcal{F}$ the operator $\hat{T}$ is defined on $I / J$ by

$$
\hat{T}(x+J)=T x+J .
$$

Because $I$ and $J$ are invariant under $T$, the operator $\hat{T}$ is a well-defined operator on $I / J$. Any such $\hat{\mathcal{F}}$ is called a collection of ideal-quotients of the family $\mathcal{F}$. A set $\mathcal{P}$ of properties is said to be inherited by ideal-quotients if every family of ideal-quotients of a family of operators satisfying properties in $\mathcal{P}$ also satisfies the same properties.

Lemma 1.2 (The Ideal-triangularization Lemma). Let $\mathcal{P}$ be a set of properties inherited by ideal-quotients. If every family of operators on a Banach lattice of dimension greater than one which satisfies $\mathcal{P}$ is ideal-reducible, then every such family is ideal-triangularizable.

Definition 1.3. An algebra $\mathcal{A}$ over a field $F$ is said to be nilpotent (resp. a nilalgebra) if there exists $n \in \mathbb{N}$ such that the product of an arbitrary $n$ elements in $\mathcal{A}$ is equal to zero (resp. if every element in $\mathcal{A}$ is nilpotent).

It is obvious that every nilpotent algebra is also a nil-algebra, but the converse statement is false in general. The following theorem of Nagata (11]) and Higman ([8]) shows the converse statement when the nilpotency index of elements is bounded.

Theorem 1.4 (Nagata-Higman). Let $\mathcal{A}$ be an algebra over a field $F$ of characteristic zero. Suppose that there is a natural number $n$ such that $a^{n}=0$ for all $a \in \mathcal{A}$. Then $a_{1} \cdots a_{2^{n}-1}=0$ for all $a_{1}, \ldots, a_{2^{n}-1} \in \mathcal{A}$.

\section{Results}

The following proposition will be used in the proof of Theorem 2.2.

Proposition 2.1. Every nilpotent algebra generated by the set of positive operators acting on a Banach lattice is ideal-triangularizable. 
Proof. Suppose that $\mathcal{A}$ is a nilpotent algebra generated by the family $\mathcal{F}$ of positive operators on a Banach lattice $E$. By the Ideal-triangularization Lemma it suffices to show that $\mathcal{A}$ is ideal-reducible. Since the $\operatorname{semigroup~} \operatorname{sg}(\mathcal{F})$ linearly spans the algebra $\mathcal{A}, \mathcal{A}$ is ideal-reducible whenever $\operatorname{sg}(\mathcal{F})$ is ideal-reducible. By the assumption, there exists $n \in \mathbb{N}$ such that $\mathcal{A}^{n}=\{0\}$ and so $\mathcal{F}^{n}=\{0\}$. Without loss of generality we may assume that $n$ is the smallest positive integer with the property $\mathcal{F}^{n}=\{0\}$. If $n=1$, then $\mathcal{F}=\{0\}$, which is obviously an ideal-triangularizable family consisting of a single operator. Thus, we may assume that $n>1$. This implies $\mathcal{F}^{n-1} \neq\{0\}$ and so there exist positive operators $A_{1}, \ldots, A_{n-1} \in \mathcal{F}$ such that $A:=A_{1} \cdots A_{n-1} \neq 0$. If $I$ is the identity operator on $E$, then $A \cdot \operatorname{sg}(\mathcal{F}) \cdot I=\{0\}$ and $\operatorname{sg}(\mathcal{F})$ is ideal-reducible by Proposition 1.1 .

The following theorem is the main theorem of this paper. Not only does it give two general results on ideal-triangularizability of nil-algebras generated by a set of positive operators, it also shows that every finitely generated nil-algebra generated by a set of positive operators has to be nilpotent. In general, there exist finitely generated nil-algebras that are not nilpotent (see [1]).

Theorem 2.2. Let $\mathcal{A}$ be an algebra generated by the set $\mathcal{F}$ of positive operators acting on a Banach lattice. Suppose that either of the following conditions holds:

(1) there exists $n \in \mathbb{N}$ such that $A^{n}=0$ for each $A$ in the convex hull of the semigroup $\operatorname{sg}(\mathcal{F})$;

(2) $\mathcal{A}$ is a finitely generated nil-algebra.

Then $\mathcal{A}$ is nilpotent and ideal-triangularizable.

Proof. To see (1) assume that $A^{n}=0$ for each $A$ in the convex hull of the semigroup $\operatorname{sg}(\mathcal{F})$. Every operator in $\mathcal{A}$ is a linear combination of operators from $\operatorname{sg}(\mathcal{F})$. Suppose that $\sum_{i=1}^{k} \lambda_{i} A_{i}$, where $\lambda_{i} \in \mathbb{R}$ and $A_{i} \in \operatorname{sg}(\mathcal{F})$, is an arbitrary operator from the algebra $\mathcal{A}$. From the assumption it follows that

$$
\left(A_{1}+\cdots+A_{k}\right)^{n}=0 .
$$

Expanding equality (2.1) and observing that every operator $A_{i}$ is positive for all $1 \leq i \leq k$, we obtain that every summand in equality (2.1) is zero. Since the summands in the expansion of $\left(\sum_{i=1}^{k} \lambda_{i} A_{i}\right)^{n}$ differ from the summands in equality (2.1) by multiplicative constants, we have

$$
\left(\lambda_{1} A_{1}+\cdots+\lambda_{k} A_{k}\right)^{n}=0 .
$$

Therefore, for every $A \in \mathcal{A}$ we have $A^{n}=0$. By Theorem 1.4 the algebra $\mathcal{A}$ is nilpotent and so it is ideal-triangularizable by Proposition 2.1.

To see (2) suppose that $\mathcal{A}$ is generated by the family $\mathcal{F}=\left\{T_{1}, \ldots, T_{n}\right\}$ of positive operators on $E$. Since every operator in $\mathcal{A}$ is nilpotent, there exists $k \in \mathbb{N}$ such that $\left(T_{1}+\cdots+T_{n}\right)^{k}=0$. We claim that an arbitrary product of $k$ operators from $\mathcal{F}$ is zero. Let $f:\{1, \ldots, k\} \rightarrow\{1, \ldots, n\}$ be any function. Then the element $T_{f(1)} \cdots T_{f(k)}$ appears as a summand in the expansion of $\left(T_{1}+\cdots+T_{n}\right)^{k}$. Since $\left(T_{1}+\cdots+T_{n}\right)^{k}=0$ and every summand in its expansion is a positive operator, it follows that $T_{f(1)} \cdots T_{f(k)}=0$. Since every operator in the algebra $\mathcal{A}$ is a linear combination of arbitrary products of operators from $\mathcal{F}$, we obtain that an arbitrary product of $k$ operators from the algebra $\mathcal{A}$ is zero and therefore $\mathcal{A}$ is a nilpotent algebra. Ideal-triangularizability of $\mathcal{A}$ again follows by Proposition 2.1. 
Corollary 2.3. Let $E$ be a Banach lattice and let $\mathcal{A}$ be a uniformly closed subalgebra of the algebra of all bounded operators on $E$. If $\mathcal{A}$ is generated by a set of positive operators and if $\mathcal{A}$ is a nil-algebra, then $\mathcal{A}$ is ideal-triangularizable.

Proof. Since $\mathcal{A}$ is a nil-Banach algebra, by [5] there exists $n \in \mathbb{N}$ such that $A^{n}=0$ for each $A \in \mathcal{A}$. Ideal-triangularizability of $\mathcal{A}$ now follows from Theorem 2.2.

Corollary 2.4. Let $\mathcal{A}$ be a nil-algebra generated by the set $\mathcal{F}$ of positive operators acting on a Banach lattice. Then any finite subset (of not necessarily positive operators) of $\mathcal{A}$ generates a nilpotent ideal-triangularizable algebra, and the algebraic tensor product $\mathcal{A} \otimes \mathcal{A}$ is a nil-algebra.

Proof. Let $\mathcal{S}$ be a finite family of operators in $\mathcal{A}$. Then there exists a finite subfamily $\mathcal{F}_{1} \subseteq \mathcal{F}$ such that $\mathcal{S}$ is contained in the algebra $\mathcal{B}$ generated by $\mathcal{F}_{1}$. By Theorem 2.2, the algebra $\mathcal{B}$ is ideal-triangularizable and nilpotent. Since the algebra generated by the family $\mathcal{S}$ is contained in the algebra $\mathcal{B}$, it has to be nilpotent and idealtriangularizable. Applying [12, Corollary 1] we obtain that the algebraic tensor product $\mathcal{A} \otimes \mathcal{A}$ is a nil-algebra.

Recall that the algebra $\mathcal{A}$ over a field $F$ is Artinian if every decreasing sequence of its two-sided ideals eventually becomes constant.

Theorem 2.5. Let $\mathcal{A}$ be an Artinian algebra generated by the family $\mathcal{F}$ of positive operators. If there exists a nonempty subfamily $\mathcal{F}_{1} \subseteq \mathcal{F}$ such that the ideal in $\mathcal{A}$ generated by $\mathcal{F}_{1}$ consists of nilpotent operators, then $\mathcal{A}$ is ideal-reducible. If $\mathcal{A}$ is a nil-algebra, then $\mathcal{A}$ is ideal-triangularizable.

Proof. To see the first part of the theorem, assume that the ideal $\mathcal{J}$ in the algebra $\mathcal{A}$ generated by a nonempty subfamily $\mathcal{F}_{1}$ of $\mathcal{F}$ consists of nilpotent operators. By Hopkins's theorem (see [7]), the ideal $\mathcal{J}$ is nilpotent. This implies that the semigroup ideal generated by the family $\mathcal{F}_{1}$ in the $\operatorname{semigroup~} \operatorname{sg}(\mathcal{F})$ is ideal-reducible, and by Proposition 1.1 it follows that $\operatorname{sg}(\mathcal{F})$ is ideal-reducible. Finally, $\mathcal{A}$ is idealreducible.

To see the second part of the theorem, observe that the algebra $\mathcal{A}$ is nilpotent and is therefore ideal-triangularizable by Proposition 2.1

In [15] Zhong constructed an irreducible multiplicative semigroup of positive nilpotent operators with unbounded nilpotency index acting on $L^{2}[0,1]$. In $[4$ the authors improved Zhong's result. They constructed an irreducible multiplicative semigroup of square-zero positive operators on $L^{p}[0,1)(1 \leq p<\infty)$ in which any finite number of elements generates an ideal-triangularizable semigroup. They even constructed an irreducible set of positive nilpotent operators on $L^{p}[0,1)$ which is closed under multiplication, addition and multiplication by positive real scalars with the property that any finite subset is ideal-triangularizable. We recall the construction from [4], as this example shows the following:

(1) in Theorem 2.2 it is not enough to assume that the convex hull of the semigroup $\operatorname{sg}(\mathcal{F})$ consists of nilpotent operators;

(2) there exists an algebra of nilpotent operators generated by the family of positive operators acting on $L^{p}[0,1)$ such that any finitely generated subalgebra is ideal-triangularizable but the whole algebra is irreducible. 
Example 2.6. Let $1 \leq p<\infty$ and let the interval $[0,1)$ be equipped with the Lebesgue measure. For $\varphi \in L^{\infty}[0,1)$, let $M_{\varphi}$ denote the multiplication operator on $L^{p}[0,1)$ defined by $\left(M_{\varphi} f\right)(x)=\varphi(x) f(x)$ for all $f \in L^{p}[0,1)$. For $\alpha \in(-1,1)$ let $U_{\alpha}$ denote the translation operator on $L^{p}[0,1)$ defined by $\left(U_{\alpha} f\right)(x)=f(x \oplus \alpha)$ for all $f \in L^{p}[0,1)$, where $\oplus$ denotes the translation modulo 1 . Let $\chi_{E}$ denote the characteristic function of the measurable subset $E \subseteq[0,1)$. Now we will construct an irreducible nil-algebra generated by the set of positive operators. Define $\mathcal{S}_{0}=\{0\}$,

$$
\mathcal{S}_{1}=\left\{0, U_{\frac{1}{2}} M_{\chi_{\left[\frac{1}{2}, 1\right)}}\right\}
$$

and similarly, for $n=2,3, \ldots$, define

$$
\mathcal{S}_{n}=\{0\} \cup\left\{U_{\frac{j}{2^{n}}} M_{\chi_{E}}: E=\left[\frac{k}{2^{n}}, \frac{k+1}{2^{n}}\right) \text { with } j, k \text { odd integers }\right\} .
$$

In [4, Theorem 2.1] it was proved that $\mathcal{S}_{n} \cdot \mathcal{S}_{n}=\{0\}$ and $\mathcal{S}_{n} \cdot \mathcal{S}_{m} \subseteq \mathcal{S}_{\max \{n, m\}}$. It follows that the set $\mathcal{S}=\bigcup_{n=0}^{\infty} \mathcal{S}_{n}$ is a countable semigroup of positive operators on $L^{p}[0,1)$. Let $\mathcal{A}$ be the algebra generated by the semigroup $\mathcal{S}$. It can be shown as in [4, Theorem 3.1] that $\mathcal{A}$ is an irreducible nil-algebra.

We conclude this paper with an application of the idea of the proof of Proposition 2.1 to obtain a different proof of [6, Theorem 4.1].

Theorem 2.7. Let $X$ be a Banach space and $\mathcal{A}$ an algebra of bounded nilpotent operators on $X$. If there exists $n \in \mathbb{N}$ such that $A^{n}=0$ for every operator $A \in \mathcal{A}$, then $\mathcal{A}$ is triangularizable.

Proof. By the Triangularization Lemma [13, 7.1.11] it suffices to show that $\mathcal{A}$ is reducible. Let $n_{0}$ be the smallest natural number such that $\mathcal{A}^{n_{0}}=\{0\}$. If $n_{0}=1$, then $\mathcal{A}=\{0\}$ and is obviously triangularizable. Otherwise we can proceed as in the proof of Theorem 2.2 to obtain a nonzero operator $T$ on $X$ such that $T A=0$ for all $A \in \mathcal{A}$. Since $\mathcal{A}$ is nonzero, there exists a nonzero vector $x \in X$ such that $Y=\mathcal{A} x \neq\{0\}$. Since $T A=0$ and $T$ is nonzero, $Y$ is not dense in $X$ and so the closure $\bar{Y}$ of $Y$ is a nontrivial closed subspace which is invariant under the algebra $\mathcal{A}$.

\section{ACKNOWLEDGEMENT}

The author is sincerely thankful to Professor Roman Drnovšek for helpful advice.

\section{REFERENCES}

[1] A. Z. Ananin, A nil algebra with nonradical tensor square, Sibirsk. Math. Zh. 26 (1985), 192-194. (Russian) MR788345 (86i:16006)

[2] R. Drnovšek, Triangularizing semigroups of positive operators on an atomic normed Riesz spaces, Proc. Edin. Math. Soc. 43 (2000), 43-55. MR1744698 (2001i:47062)

[3] R. Drnovšek, M. Kandić, Ideal-triangularizability of semigroups of positive operators, Integral Equations and Operator Theory 64 (2009), no. 4, 539-552. MR.2534032

[4] R. Drnovšek, D. Kokol-Bukovšek, L. Livshits, G. MacDonald, M. Omladič, H. Radjavi, An irreducible semigroup of non-negative square-zero operators, Integral Equations and Operator Theory 42 (2002), no. 4, 449-460. MR.1885443 (2003j:47051)

[5] S. Grabiner, The nilpotency of Banach nil algebras, Proc. Amer. Math. Soc. 21 (1969), 510. MR0236700 (38:4995)

[6] D. W. Hadwin, E. A. Nordgren, M. Radjabalipour, H. Radjavi, P. Rosenthal, On simultaneous triangularization of collections of operators, Houston J. Math. 17 (1991), no. 5, 581-602. MR:1147275 (93a:47004) 
[7] I. Herstein, Noncommutative rings, Carus Mathematical Monographs 15, Mathematical Association of America (2005). MR1449137 (97m:16001)

[8] G. Higman, On a conjecture of Nagata, Proc. Camb. Phil. Soc. 52 (1956), 1-4. MR0073581 $(17: 453 \mathrm{e})$

[9] M. T. Jahandideh, On the ideal-triangularizability of positive operators on Banach lattices, Proc. Amer. Math. Soc. 125 (1997), 2661-2670. MR1396983 (97j:47052)

[10] W. A. J. Luxemburg, A. C. Zaanen, Riesz spaces. I, North Holland, Amsterdam, 1971. MR0511676 (58:23483)

[11] M. Nagata, On the nilpotency of nil-algebras, J. Math. Soc. Japan 4 (1952), 296-301. MR0053088 (14:719g)

[12] E. R. Puczylowski, Nil algebras with nonradical tensor square, Proc. Amer. Math. Soc. 103 (1988), 401-403. MR943055 (89e:16021)

[13] H. Radjavi, P. Rosenthal, Simultaneous triangularization, Universitext, Springer-Verlag, New York, 2000. MR,1736065 (2001e:47001)

[14] H. H. Schaefer, Banach Lattices and Positive Operators, Die Grundlehren der Mathematischen Wissenschaften 215, Springer-Verlag, Berlin-Heidelberg-New York, 1974. MR0423039 $(54: 11023)$

[15] Y. Zhong, Irreducible semigroups of functionally positive nilpotent operators, Trans. Amer. Math. Soc. 347 (1995), 3093-3100. MR1264835 (95j:47008)

Institute of Mathematics, Physics and Mechanics, Jadranska 19, 1000 Luubluana, SLOVENIA

E-mail address: marko.kandic@fmf.uni-lj.si 